\title{
Some Properties and Applications of the Riemann- Hadamard Function of Darboux Problem for Telegraph Equation
}

\author{
Akimov Andrey ${ }^{1}$ \\ Sterlitamak, Russia \\ Bashkir state university Sterlitamak branch \\ 453103, Lenina street 47A
}

\begin{abstract}
The article deals with the problem of constructing solution of the Darboux problem for telegraph equation for the case with deviation from the characteristic. In this paper preliminarily is constructed RiemannHadamard function and uniqueness theorem is established for Darboux problem. Then using the function of Riemann-Hadamard was constructed a solution of the Darboux problem explicitly.
\end{abstract}

Keywords: Darboux problem, the Riemann-Hadamard function, telegraph equation

\section{INTRODUCTION AND PRELIMINARY}

Consider a problem of the Darboux type for the telegrath equation

$L_{0} v=v_{x x}-v_{y y}+c v=0$,

where $c$ is an arbitrary complex number in the domain $D$ bounded by the characteristic $C B(x+y=1)$ of equation (1), line $A C(k x-y=0)$, and by the segment $A B$ of the axis $y=0$.

Problem $D$. In the domain $D$ find a function $v(x, y)$ which satisfies the conditions:

$$
\begin{aligned}
& v(x, y) \in C(\bar{D}) \wedge C^{1}(D \cup A B) \wedge C^{2}(D) ; \\
& L v(x, y) \equiv 0, \quad(x, y) \in D ; \\
& v(x, 0)=\tau(x), \quad 0<x<1 ; \\
& v(x, k x)=\varphi(x), \quad 0<x<\frac{1}{1+k},
\end{aligned}
$$

where $\tau(x)$ and $\phi(x)$ are given sufficiently smooth functions.

Definition. We call a function $v(x, y)$ quazi-regular solution of (1) if the following hold

- $v(x, y)$ satisfies (2);

- we can to applicate Green's theorem to the integrals

$$
\iint_{D} v L_{0} v d x d y, \quad \iint_{D} v_{x} L_{0} v d x d y, \quad \iint_{D} v_{y} L_{0} v d x d y
$$

- the boundary integrals which arise exist in the sense that: the limits taken over corresponding interior curves exist as these interior curves approach the boundary.

\section{THEOREM OF UNIQUENESS}

Assume $v_{1}, v_{2}$ : two solutions of Problem $D$ for equation (1) and boundary conditons. Then take $v=v_{1}-v_{2}$. In this case function $v(x, y)$ in $D$ satisfying equation (1) and following boundary conditions

\footnotetext{
${ }^{1}$ Corresponding Author: andakm@ rambler.ru
} 
American Research Journal of Mathematics, Volume 1, Issue 3, June 2015

ISSN 2378-704X

$$
\left\{\begin{array}{c}
v=0 \text { on } \gamma_{1} \\
v=0 \text { on }\{y=0\} \cap D
\end{array}\right.
$$

Therefore for proof uniqueness solution Problem $D$ it is enough to show that $v=0$ in $D$.

Theorem 1. If $v(x, y)$ - quazi-regular solution of (1) in $D$ and constant $c>0,\left.\quad v\right|_{\{\{y=0\} \cap D\} \cup \gamma_{1}}=0, \quad$ then $v(x, y) \equiv 0$ in $D$.

Proof. Consider $v=v(x, y)$ be a quazi-regular of (1) defined in $D$. Besides consider the integral

$$
2 \iint_{D}\left(b v_{x}+a v_{y}\right)\left(v_{x x}-v_{y y}+c v\right) d x d y
$$

where $a, b$ sufficiently smooth functions of $(x, y)$.

By virtue of (1) this integral vanishes. The functions $a, b$ are chosen in in such a way that, after a transformation of the integral by Green's formula, one obtains a positive (or non-negative) definite expression which vanishes only if $v=0$ in $D$.

Consider identities

$$
\begin{gathered}
b v_{x} v_{x x}=\frac{1}{2}\left(b v_{x}^{2}\right)_{x}-\frac{1}{2} b_{x} v_{x}^{2}, \\
a v_{y} v_{y y}=\frac{1}{2}\left(a v_{y}^{2}\right)_{y}-\frac{1}{2} a_{y} v_{y}^{2}, \\
a v_{y} v_{x x}=\left(a v_{x} v_{y}\right)_{x}-\frac{1}{2}\left(a v_{x}^{2}\right)_{y}+\frac{1}{2} a_{y} v_{x}^{2}-a_{x} v_{x} v_{y}, \\
b v_{x} v_{y y}=\left(b v_{x} v_{y}\right)_{y}-b_{y} v_{x} v_{y}-\frac{1}{2}\left(b v_{y}^{2}\right)_{x}+\frac{1}{2} b_{x} v_{y}^{2}, \\
b v_{x} v=\frac{1}{2}\left(b v^{2}\right)_{x}-\frac{1}{2} b_{x} v^{2}, \\
a v_{y} v=\frac{1}{2}\left(v^{2}\right)_{y}-\frac{1}{2} a_{y} v^{2}
\end{gathered}
$$

Besides employ Green's theorem:

$$
\int_{\partial D} P(x, y) d x+Q(x, y) d y=\iint_{D}\left(\frac{\partial Q}{\partial x}-\frac{\partial P}{\partial y}\right) d x d y .
$$

Then employing above identities and applying Greene theorem we get:

$$
\begin{gathered}
J=\iint_{D}\left(b_{x} v_{x}^{2}-a_{y} v_{x}^{2}+2 a_{x} v_{x} v_{y}+b_{x} v_{y}^{2}+b_{x} v^{2}+a_{y} v^{2}\right) d x d y+ \\
+\int_{\gamma_{1}}+\int_{\gamma_{2}}+\int_{\{y=0\} \cap D}\left(b v_{x}^{2}+2 a v_{x} v_{y}+b v_{y}^{2}+b c v^{2}\right) d y+\left(a v_{y}^{2}+2 b v_{x} v_{y}+a v_{x}^{2}-a c v^{2}\right) d x= \\
=I+J_{1}+J_{2}+J_{3} \\
0=\int_{\gamma_{1} \cup \gamma_{2} \cup\{y=0\}}\left(b v_{x}^{2}+2 a v_{x} v_{y}+b v_{y}^{2}+b c v^{2}\right) d y+\left(a v_{y}^{2}+2 b v_{x} v_{y}+a v_{x}^{2}-a c v^{2}\right) d x=J_{1}+J_{2}+J_{3}
\end{gathered}
$$

Finally we must choose: "nice functions" $a=a(x, y), b=b(x, y)$, in $D$ so that all conditions hold. If this occurs then uniqueness follows immediately. 
Choose $a=-k b, b=c$. Obviously $I=0$. From $v(x, y)=0$ on $\{y=0\} \cup \gamma_{1}$ and the fact that

$$
d y=-k d x\left(\text { on } \gamma_{1}\right), d y=d x\left(\text { on } \gamma_{2}\right)
$$

we get

$$
\begin{gathered}
d y=-k d x \quad v=0 \quad v_{y} d y+v_{x} d x=0 \\
J_{1}=\int_{\gamma_{1}}(k b+a)\left(1-k^{2}\right)\left(v_{y}^{2}-c v^{2}\right) d x=0 \\
J_{2}=\int_{\gamma_{2}}\left[c(1-k)\left(v_{x}+v_{y}\right)^{2}+c^{2} v^{2}(1+k)\right] d x \geq 0 \\
J_{3}=-k c \int_{\{y=0\} \cap D} v_{y}^{2} d x \geq 0
\end{gathered}
$$

\section{CONSTRUCTION FUNCTION RIEMANN-HADAMARD}

On the plane $(x, y)$ we pass to the characteristic coordinates $\xi=x+y, \eta=x-y$. Then equation (1) takes the form

$$
L u=u_{\xi \eta}+\frac{c}{4} u=0
$$

where

$$
u(\xi, \eta)=u\left(\frac{1}{2}(\xi+\eta), \frac{1}{2}(\xi-\eta)\right)
$$

and the domain $D$ is mapped to the domain

$$
\Delta=\left\{(\xi, \eta) \mid 0<\xi<\eta<\alpha \xi<1, \alpha=\frac{1-k}{1+k}>1\right\}
$$

and, respectively, Problem $D$ is posed as follows:

Problem $D^{\prime}$. In the domain $\Delta$ find a function $v(x, y)$ which satisfies the conditions

$$
\begin{gathered}
u(\xi, \eta) \in C(\bar{\Delta}) \wedge C^{1}(\Delta \cup\{\eta=\alpha \xi\}) \wedge u_{\xi \eta} \in C(\Delta) ; \\
L u(\xi, \eta) \equiv 0,(\xi, \eta) \in \Delta, \\
u(\xi, \alpha \xi)=\tau(\xi), 0 \leq \xi \leq \frac{1}{\alpha} \\
u(\xi, \xi)=\psi(\xi), 0 \leq \xi \leq 1 ; \\
\psi(0)=\varphi(0) .
\end{gathered}
$$

It is well known that the Riemann--Hadamard function plays an important role in the study of problem $D^{\prime}$; this function was defined and constructed in [1-6] for some special cases of Eq.(1).In this section, we present an in a sense modified (as compared with the approaches used in the above-mentioned papers) approach to defining the Riemann--Hadamard function of problem $D^{\prime}$ for Eq. (1) in case if boundary values is defined on non-characteristic.

Let domain $\Delta$ is divided into following subdomains

$$
\begin{gathered}
\sigma_{0}=\left\{(\xi, \eta) \mid \eta<\alpha \xi, \xi<\frac{\eta_{0}}{\alpha}, \eta>\xi_{0}\right\}, \\
\sigma_{2 k}=\left\{(\xi, \eta) \mid \eta<\alpha \xi, \xi<\frac{\eta_{0}}{\alpha^{k+1}}, \eta>\frac{\xi_{0}}{\alpha^{k}}\right\},
\end{gathered}
$$




$$
\begin{gathered}
\sigma_{2 k+1}=\left\{(\xi, \eta) \mid \eta<\alpha \xi, \xi<\frac{\xi_{0}}{\alpha^{k+1}}, \eta>\frac{\eta_{0}}{\alpha^{k+1}}\right\}, \\
\omega_{0}=\left\{(\xi, \eta) \mid \eta<\eta_{0}, \xi<\xi_{0}, \eta>\xi_{0}, \xi>\frac{\eta_{0}}{\alpha}\right\}, \\
\omega_{2 k}=\left\{(\xi, \eta) \mid \xi>\frac{\eta_{0}}{\alpha^{k+1}}, \xi<\frac{\xi_{0}}{\alpha^{k}}, \eta<\frac{\eta_{0}}{\alpha^{k}}, \eta>\frac{\xi_{0}}{\alpha^{k}}\right\}, \\
\omega_{2 k+1}=\left\{(\xi, \eta) \mid \xi<\frac{\eta_{0}}{\alpha^{k+1}}, \xi>\frac{\xi_{0}}{\alpha^{k+1}}, \eta>\frac{\eta_{0}}{\alpha^{k+1}}, \eta<\frac{\xi_{0}}{\alpha^{k}}\right\}, \\
\Delta_{2 k+1}=\left\{(\xi, \eta) \mid \eta>\xi, \xi>\frac{\eta_{0}}{\alpha^{k+1}}, \eta<\frac{\xi_{0}}{\alpha^{k}}\right\}, \\
\Delta_{2 k}=\left\{(\xi, \eta) \mid \eta>\xi, \xi>\frac{\xi_{0}}{\alpha^{k}}, \eta<\frac{\eta_{0}}{\alpha^{k}}\right\}, \\
k=1,2, \ldots . .
\end{gathered}
$$

In what follows, we assume that function is known as the Riemann-Hadamard function $R\left(\xi, \eta ; \xi_{0}, \eta_{0}\right)$ satisfies conditions

$$
\begin{gathered}
1 . L R\left(\xi, \eta ; \xi_{0}, \eta_{0}\right)=R_{\xi \eta}+c R=0 . \\
\text { 2. }\left.R_{\xi}\right|_{\eta=\eta_{0}}=0,\left.\quad R_{\eta}\right|_{\xi=\xi_{0}}=0,\left.\quad R\right|_{\eta=\xi \cup \eta=\alpha \xi}=0 . \\
3 . \frac{\partial\left[R_{1}\right]}{\partial \xi}=0,\left[R_{1}\right]=\lim _{\varepsilon \rightarrow 0}\left[R\left(\xi ; \frac{\xi_{0}}{\alpha^{k-1}}+\varepsilon ; \xi_{0} ; \eta_{0}\right)-R\left(\xi ; \frac{\xi_{0}}{\alpha^{k-1}}-\varepsilon ; \xi_{0} ; \eta_{0}\right)\right] \\
\frac{\partial\left[R_{2}\right]}{\partial \xi}=0,\left[R_{2}\right]=\lim _{\varepsilon \rightarrow 0}\left[R\left(\xi ; \frac{\eta_{0}}{\alpha^{k}}+\varepsilon ; \xi_{0} ; \eta_{0}\right)-R\left(\xi ; \frac{\eta_{0}}{\alpha^{k}}-\varepsilon ; \xi_{0} ; \eta_{0}\right)\right] \\
\frac{\partial\left[R_{3}\right]}{\partial \eta}=0,\left[R_{3}\right]=\lim _{\varepsilon \rightarrow 0}\left[R\left(\frac{\xi_{0}}{\alpha^{k}}+\varepsilon ; \eta ; \xi_{0} ; \eta_{0}\right)-R\left(\frac{\xi_{0}}{\alpha^{k}}-\varepsilon ; \eta ; \xi_{0} ; \eta_{0}\right)\right] \\
\frac{\partial\left[R_{4}\right]}{\partial \xi}=0,\left[R_{4}\right]=\lim _{\varepsilon \rightarrow 0}\left[R\left(\frac{\eta_{0}}{\alpha^{k}}+\varepsilon ; \xi \xi_{0} ; \eta_{0}\right)-R\left(\frac{\eta_{0}}{\alpha^{k}}-\varepsilon ; \xi ; \xi_{0} ; \eta_{0}\right)\right] \\
4 . R\left(\xi, \eta, \xi_{0}, \eta_{0}\right)=1 \\
k=1,2, \ldots . .
\end{gathered}
$$

Then the function of the Riemann-Hadamard is determined by the recurrent formulas as follows

$$
\begin{gathered}
R_{\sigma_{2 k}}=R_{\omega_{2 k}}-J_{0}\left(\sqrt{c\left(\eta \alpha^{-k-1}-\xi_{0}\right)\left(\alpha^{k+1} \xi-\eta_{0}\right)}\right),(\xi, \eta) \in \sigma_{2 k}, \\
\left.R_{\sigma_{2 k+1}}=R_{\omega_{2 k+1}}+J_{0}\left(\sqrt{c\left(\alpha^{k+1} \xi-\xi_{0}\right)\left(\eta \alpha^{-k-1}-\eta_{0}\right.}\right)\right),(\xi, \eta) \in \sigma_{2 k+1}, \\
\left.R_{\Delta_{2 k+1}}=R_{\omega_{2 k}}-J_{0}\left(\sqrt{c\left(\alpha^{k} \eta-\xi_{0}\right)\left(\xi \alpha^{-k}-\eta_{0}\right.}\right)\right),(\xi, \eta) \in \Delta_{2 k+1}, \\
\left.R_{\Delta_{2 k+2}}=R_{\omega_{2 k+1}}+J_{0}\left(\sqrt{c\left(\alpha^{k+1} \eta-\eta_{0}\right)\left(\xi \alpha^{-k-1}-\xi_{0}\right.}\right)\right),(\xi, \eta) \in \Delta_{2 k+2},
\end{gathered}
$$


American Research Journal of Mathematics, Volume 1, Issue 3, June 2015

ISSN 2378-704X

$$
\begin{aligned}
& \left.R_{\omega_{2 k+1}}=R_{\Delta_{2 k+1}}-J_{0}\left(\sqrt{c\left(\alpha^{k+1} \xi-\eta_{0}\right)\left(\eta \alpha^{-k-1}-\xi_{0}\right.}\right)\right),(\xi, \eta) \in \omega_{2 k+1}, \\
& \left.R_{\omega_{2 k+2}}=R_{\Delta_{2 k+2}}-J_{0}\left(\sqrt{c\left(\alpha^{k+1} \xi-\xi_{0}\right)\left(\eta \alpha^{-k-1}-\eta_{0}\right.}\right)\right),(\xi, \eta) \in \omega_{2 k+2},
\end{aligned}
$$

where $J_{0}(\cdot)$ is Bessel function of zero order.

\section{Construction Solution OF DARboux Problem}

One can readily see that

$u \cdot L R-R \cdot L u=\frac{1}{2}\left(u R_{\eta}-R u_{\eta}\right)_{\xi}+\frac{1}{2}\left(u R_{\xi}-R u_{\xi}\right)_{\eta}$

By using relation (6), where $u$ is a regular solution of Eq. (1) in the domain $\Delta$ and $R$ is function of RiemannHadamard, and by applying the Green formula to the above-mentioned subdomains $\omega_{k}, \sigma_{k}, \Delta_{k}$ of the domain $\Delta$, one can readily justify the relations

$$
\begin{gathered}
0=\int_{\left(\cup \partial \Delta_{i} \cup \partial \omega_{k} \cup \partial \sigma_{m}\right)}\left(u R_{\xi}-R u_{\xi}\right) d \xi-\left(u R_{\eta}-R u_{\eta}\right) d \eta= \\
=I_{E D}+I_{D C}+I_{C A}+I_{A E},
\end{gathered}
$$

where $D=\left(\xi_{0}, \eta_{0}\right), C=\left(\frac{\eta_{0}}{\alpha}, \eta_{0}\right), B=\left(\frac{\xi_{0}}{\alpha}, \xi_{0}\right), A=(0,0), E=\left(\xi_{0}, \xi_{0}\right)$.

Calculating the integrals $I_{E D}, I_{D C}, I_{C A}, I_{A E}$, one obtains:

$$
\begin{gathered}
I_{E D}=\left.u R_{1}\right|_{E} ^{D}=u R_{1}(D)-u R_{1}(E)=u\left(\xi_{0}, \eta_{0}\right)-\tau\left(\xi_{0}\right) \\
I_{D C}=-\left.u R_{1}\right|_{D} ^{C}=-u R_{1}(C)+u R_{1}(D)=-\psi\left(\frac{\eta_{0}}{\alpha}\right)+u\left(\xi_{0}, \eta_{0}\right)
\end{gathered}
$$

Since $d \eta=\alpha d \xi$ on $A C$ then

$$
\begin{aligned}
& I_{A C}=\int_{0}^{\frac{\eta_{0}}{\alpha}} u\left[\left(R_{\xi}-\alpha\left(R_{\eta}\right] d \xi=\right.\right.
\end{aligned}
$$

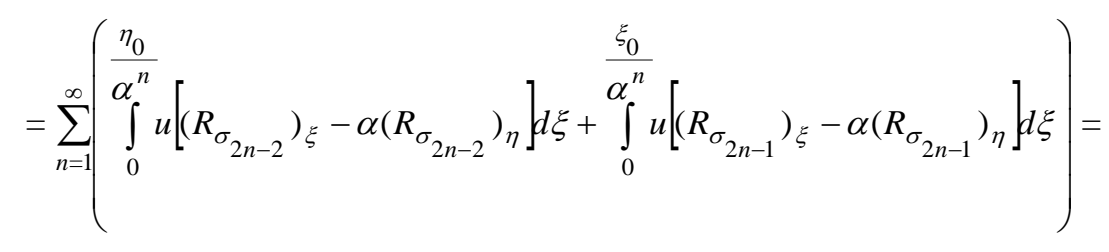

$$
\begin{aligned}
& =\sum_{n=1}^{\infty} A\left(\eta_{0}-\alpha^{2 n-1} \xi_{0}\right)^{\frac{\eta_{0}}{\alpha^{n}}} \int_{0} \frac{\left.J_{1}\left(\sqrt{c\left(\alpha^{n} \xi-\eta_{0}\right)\left(\xi \alpha^{1-n}-\xi_{0}\right.}\right)\right)}{\left.\sqrt{\left(\alpha^{n-1} \xi-\alpha^{2 n-2} \xi_{0}\right)\left(\alpha^{n} \xi-\eta_{0}\right.}\right)} \tau(\xi) d \xi+ \\
& +\sum_{n=1}^{\infty} A\left(\alpha^{2 n-1} \eta_{0}-\xi_{0}\right) \int_{0}^{\frac{\xi_{0}}{\alpha^{n}}} \frac{\left.J_{1}\left(\sqrt{c\left(\alpha^{n} \xi-\xi_{0}\right)\left(\xi \alpha^{1-n}-\eta_{0}\right.}\right)\right)}{\sqrt{\left(\alpha^{n-1} \xi-\alpha^{2 n-2} \eta_{0}\right)\left(\alpha^{n} \xi-\xi_{0}\right)}} \tau(\xi) d \xi
\end{aligned}
$$

Again, on $A E, d \eta=d \xi$. Hence 


$$
\begin{aligned}
& I_{A E}=\int_{0}^{\xi_{0}} u\left(R_{\xi}-R_{\eta}\right) d \xi=\int_{0}^{\xi_{0}} u\left[\left(R_{\Delta_{1}}\right)_{\xi}-\left(R_{\Delta_{1}}\right)_{\eta}\right] d \xi+
\end{aligned}
$$

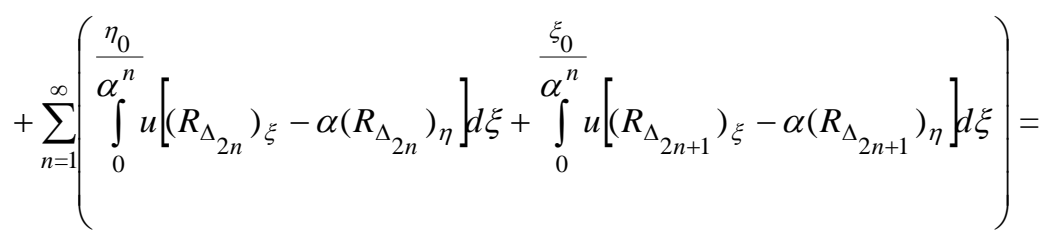

$$
\begin{aligned}
& =A(\eta-\xi) \int_{0}^{\xi} \frac{J_{1}(\sqrt{c(t-\xi)(t-\eta)})}{\sqrt{(t-\xi)(t-\eta)}} \psi(t) d t+ \\
& +\sum_{n=1}^{\infty} A\left(\alpha^{2 n} \xi_{0}-\eta_{0}\right) \int_{0}^{\frac{\eta_{0}}{\alpha^{n}}} \frac{J_{1}\left(\sqrt{c\left(\alpha^{n} \xi-\eta_{0}\right)\left(\xi \alpha^{-n}-\xi_{0}\right.}\right)}{\left.\sqrt{\left(\alpha^{n} \xi-\alpha^{2 n} \xi_{0}\right)\left(\alpha^{n} \xi-\eta_{0}\right.}\right)} \psi(\xi) d \xi+ \\
& +\sum_{n=1}^{\infty} A\left(\xi_{0}-\alpha^{2 n} \eta_{0}\right)^{\frac{\xi_{0}}{\alpha^{n}}} \frac{\left.J_{1}\left(\sqrt{c\left(\alpha^{n} \xi-\xi_{0}\right)\left(\xi \alpha^{-n}-\eta_{0}\right.}\right)\right)}{\left.\sqrt{\left(\alpha^{n} \xi-\alpha^{2 n} \eta_{0}\right)\left(\alpha^{n} \xi-\xi_{0}\right.}\right)} \psi(\xi) d \xi
\end{aligned}
$$

Therefore, one can readily show that the solution of the problem $D^{\prime}$ can be represented at the point $(\xi, \eta) \in \Delta$ in the form

$$
\begin{aligned}
& u(\xi, \eta)=\tau(\xi)+\psi\left(\frac{\eta}{\alpha}\right)+A(\eta-\xi) \int_{0}^{\xi} \frac{J_{1}(\sqrt{c(t-\xi)(t-\eta)})}{\sqrt{(t-\xi)(t-\eta)}} \psi(t) d t+ \\
& +\sum_{n=1}^{\infty} A\left(\eta-\alpha^{2 n-1} \xi\right) \int_{0}^{\frac{\eta}{\alpha^{n}}} \frac{J_{1}\left(\sqrt{c\left(\alpha^{n} t-\eta\right)\left(t \alpha^{1-n}-\xi\right)}\right)}{\sqrt{\left(\alpha^{n-1} t-\alpha^{2 n-2} \xi\right)\left(\alpha^{n} t-\eta\right)}} \tau(t) d t+ \\
& +\sum_{n=1}^{\infty} A\left(\alpha^{2 n-1} \eta-\xi\right) \int_{0}^{\frac{\xi}{\alpha^{n}}} \frac{J_{1}\left(\sqrt{c\left(\alpha^{n} t-\xi\right)\left(t \alpha^{1-n}-\eta\right)}\right)}{\sqrt{\left(\alpha^{n-1} t-\alpha^{2 n-2} \eta\right)\left(\alpha^{n} t-\xi\right)}} \tau(t) d t+ \\
& +\sum_{n=1}^{\infty} A\left(\alpha^{2 n} \xi-\eta\right)^{\alpha^{n}} \frac{J_{1}\left(\sqrt{c\left(\alpha^{n} t-\eta\right)\left(t \alpha^{-n}-\xi\right)}\right)}{\sqrt{\left(\alpha^{n} t-\alpha^{2 n} \xi\right)\left(\alpha^{n} t-\eta\right)}} \psi(t) d t+ \\
& +\sum_{n=1}^{\infty} A\left(\xi-\alpha^{2 n} \eta\right)^{\frac{\xi}{\alpha^{n}}} \frac{J_{1}\left(\sqrt{c\left(\alpha^{n} t-\xi\right)\left(t \alpha^{-n}-\eta\right)}\right)}{\sqrt{\left(\alpha^{n} \xi-\alpha^{2 n} \eta\right)\left(\alpha^{n} t-\xi\right)}} \psi(t) d t
\end{aligned}
$$

Theorem 2. 1If functions $\tau(\xi) \in C^{1}\left[0, \frac{1}{\alpha}\right], \alpha>1, \psi(\xi) \in C^{2}[0,1]$ and $c>0$ there exist a uniqueness solution to the problem $D^{\prime}$ of the form (7).

\section{COnClusions}

In this article, we discussed a little-known method of constructing the Riemann-Hadamard function for telegraph equation for the case with deviation from the characteristic. This result is a new study on the issue. 
American Research Journal of Mathematics, Volume 1, Issue 3, June 2015

ISSN 2378-704X

\section{REFERENCES}

[1] Akimov A.A., On uniqueness Morawetz problem for the Chaplygin equation // IJPAM, vol. 97, No. 3, (2014).

[2] Akimov Andrey, Kazakova Yevgeniya, Vildyaeva Anzhelika, Construction of the Solution of the Caushy's Problem by the Riemann's Method for a Hyperbolic Equation, American Research Journal of Mathematics, Vol. 1, Issue 2, 44-48, (2015).

[3] J.S. Papadakis †, D.H. Wood, An addition formula for Riemann functions Journal of differential Equations, 24, (1977), 397411.

[4] E.T. Copson, On the Riemann-Green Function, J. Rat.Mech. Anal., 1, (1958), 324-348.

[5] H.Wood, Simple Riemann functions, Bull. Amer. Math. Soc. vol. 82, No. 5, (1976), 737-739.

[6] M.M. Smirnov, Equations of mixed type, American Mathematical Society, United States, (1978).

[7] Courant R.,Hilbert D., Methods of Mathematical Physics, Interscience Publishers, Inc., New York, (1953).

[8] K.B. Sabitov and R.R. Il'yasov Ill-posedness of boundary value problems for one class of hyperbolic equations, Russian Mathematics(Iz. VUZ), vol. 45, No. 5, (2001), 56-60.

[9] A.G Mackie, Green's function and Riemann's method Proc. Edinburgh Math. Soc., 14, 1964, 293-302

[10] Lerner, M.E., Qualitative Properties of the Riemann Function, Differ. Uravn., , vol. 27, No. 12, (1991), 2106-2120.

[11] Bitsadze, A.V., Uravneniya matematicheskoi fiziki (Equations of Mathematical Physics), Moscow: Nauka, 1976. 